FORMATION Formation emploi

Revue française de sciences sociales

119 | juillet-septembre 2012

pêle-mêle

\title{
La non-qualification : révélateur ultime ou masque multiforme de la nature du travail ? Recension de l'ouvrage de José Rose, Qu'est-ce que le travail non qualifié?
}

\section{Robert Pierron}

\section{OpenEdition}

\section{Journals}

Édition électronique

URL : http://journals.openedition.org/formationemploi/3747

DOI : 10.4000/formationemploi.3747

ISSN : $2107-0946$

Éditeur

La Documentation française

Édition imprimée

Date de publication : 10 septembre 2012

ISSN : 0759-6340

\section{Référence électronique}

Robert Pierron, «La non-qualification : révélateur ultime ou masque multiforme de la nature du

travail ? Recension de l'ouvrage de José Rose, Qu'est-ce que le travail non qualifié ? ", Formation emploi [En ligne], 119 | juillet-septembre 2012, mis en ligne le 26 octobre 2012, consulté le 30 octobre 2020. URL : http://journals.openedition.org/formationemploi/3747 ; DOI : https://doi.org/10.4000/ formationemploi.3747 


\title{
Note de lecture
}

\section{La non-qualification : révélateur ultime ou masque multiforme de la nature du travail ?}

\author{
Recension de l'ouvrage de José Rose \\ Qu'est-ce que le travail non qualifié?
}

par Robert PIERRON

Economiste, conseiller technique CPRDFP (Contrat de plan régional de développement des formations professionnelles) au conseil régional d'Aquitaine et chercheur associé à I'IEP

(Institut d'études politiques) de Bordeaux

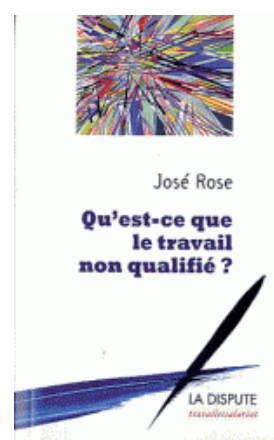

Peut-on présenter un ouvrage dont le titre prend la forme d'une question dans un texte ayant pour titre une autre question, et en introduisant ainsi une sorte de halo d'incertitude autour d'un objet lui-même particulièrement conjectural ?

En répondant par l'affirmative à une telle interrogation, on considère que ne valent vraiment comme sujets d' investigation que ceux dont l'élucidation n'est pas évidente et qui impliquent par conséquent un jeu de miroirs entre l'ambition de contribuer à résoudre un problème capital et les résultats inévitablement toujours fragmentaires et provisoires auxquels il est possible de parvenir dans une telle entreprise.

En fait, c'est bien le premier mérite de l'ouvrage que publie José Rose sur un sujet - et justement, en fait, une question - dont la récurrence et finalement la permanence s'affirment, que de réinterroger la place du travail non qualifié par delà toutes les réponses données depuis des décennies, en affirmant en fait que ce type de travail devait, allait ou aurait dû inéluctablement reculer et disparaître.

Avec des présupposés divers, s'était imposée, en effet, l'idée selon laquelle il ne s'agissait en somme, avec le travail non qualifié, en forçant le trait, que d'une sorte de relique d'un mal voué à l'éradication ; à savoir l'arriération archaïque et coupable des systèmes du travail, de l'emploi et de la formation, appelés à se transformer sous la poussée du progrès économique et social. 
Or, si la question demeure, c'est que le phénomène du travail non qualifié (TNQ) n'a pas disparu et c'est bien de cela qu'il convient de repartir, conformément à l'obstination nécessaire et inhérente à la recherche scientifique. Si, selon l'adage bien connu, «les faits sont têtus ", les chercheurs se doivent d'être plus têtus encore. C'est pourquoi l'ouvrage de José Rose apparaît comme particulièrement opportun, après différents moments de l'évolution de la place de la non-qualification dans les sociétés contemporaines; et ce en regard tout à la fois des observations précises qu'ont permis les moments de ces évolutions depuis une vingtaine d'années et les avancées interprétatives et théoriques que ces observations ont rendu possibles.

C'est ainsi que cinq éléments apparaissent spécialement significatifs dans l'ensemble de la littérature, avec pour chacun d'eux, la mention d'une publication ayant marqué une étape notable dans l'analyse du TNQ:

Tout d'abord, la persistance du TNQ semble étrange en regard des conditions quantitatives et qualitatives de la forte recréation d'emplois de la fin des années 1990, particulièrement en 1999-2000, période comparable aux meilleures années des Trente Glorieuses en termes de relation croissance-emploi. On aurait pu penser que le bilan de cette période aurait pu ou dû s'accompagner de « leçons » tirées des périodes de difficultés antérieures et de l'ardente injonction à former et à qualifier, fortement exprimée en France, à travers des alternances politiques rapprochées, en une forte continuité, notamment sensible à travers des lois importantes, promulguées de 1987 à 1993. Ce n'est d'ailleurs pas par hasard si l'ouvrage collectif le plus significatif paru sur la question du TNQ a été publié au sortir de ces années paradoxales (Méda, Vennat, (eds.), 2005).

Concomitamment, cette persistance en impliquait une autre pour les chercheurs travaillant sur les relations formation-emploi, à travers le constat du fait brutal et paradoxal de la difficulté de caractériser ces relations dans le cas des emplois non qualifiés (ENQ), dont l'hétérogénéité rendait, en période de croissance aussi bien forte que faible, inaccessible la référence à une caractérisation unique des termes de ces relations (Béduwé, 2005).

Ensuite et en quelque sorte " en creux ", cette inaccessibilité pour ainsi dire aggravée des relations formation-emploi, venait impacter rétrospectivement les idées dominantes développées au cours des années 1980 et 1990 ; ces idées avaient tendu à établir un système de l'emploi fondé sur l'enchaînement d'un cercle vertueux, capable d'impulser une nouvelle croissance par un enchaînement de l'accélération de l'innovation technologique et de l'intensité des efforts de formation, impliquant l'une et l'autre une élévation des qualifications, conformément à un modèle adopté dans de nombreux pays (Tremblay, dir., 1996).

De façon parallèle, le constat du maintien des ENQ à un niveau conséquent dans les systèmes d'emploi avait débouché sur une mobilisation de ceux-ci dans les politiques publiques d'insertion, en établissant ce qui semblait être un sas providentiel pour conduire dans l'emploi ceux qui semblaient en être les plus éloignés. Malheureusement, les démonstrations n'allaient pas tarder, étayées par des analyses statistiques rigoureuses, 
qui conduiraient à faire craindre un « effet de trappe » aussi violent que classique pour les économistes : aider les ENQ conduisait à dualiser le marché du travail et à maintenir dans ces emplois des personnes ayant finalement peu de possibilités d'accéder à la qualification (Bélani, 2007).

Enfin, de manière plus fondamentale, il n'est pas exagéré de voir dans la question des ENQ un aspect majeur des relations d'emploi et surtout de tout ce qui, dans celles-ci, ne peut relever du modèle standard. Les ENQ seraient alors l'un des symptômes majeurs d'un découplage impossible à occulter entre les structures de l'emploi et celles des qualifications. Un tel fait structurel ne pourrait pas ne pas s'accompagner d'un aggiornamento théorique obligeant à revisiter d'autres approches que celles de l'orthodoxie en matière d'analyse du marché du travail comme sur d'autres champs (Harribey, 2010).

Ces cinq éléments sont exposés et se retrouvent à plusieurs reprises dans la synthèse et les mises en perspective opérées par José Rose ; et ce, tant à travers le constat de la pluralité des aspects de ce dont on parle en évoquant la non-qualification (1), qu'en étant conduit à constater la difficulté de faire de la baisse des ENQ un objectif réellement prioritaire des politiques publiques (2).

Devrait-on alors en conclure que la question de la nature du TNQ cache sans doute bien d'autres interrogations (3), en reconnaissant d'abord qu'un phénomène structurel devrait appeler un traitement lui-même structurel ?

\section{Le fil directeur : à la poursuite de la « non-qualification » et de ses divers aspects}

L'ouvrage vaut en tout premier lieu par sa méthode analytique, consistant à aborder la question de la non-qualification sous différents angles et de façon conceptuelle, en évitant d'encombrer le texte par de nombreuses données statistiques, auxquelles le lecteur est cependant renvoyé par des références précises aux travaux et sources disponibles pour mesurer et comprendre le phénomène étudié.

De ce fait, il présente la caractéristique, assez rare pour qu'on la signale, d'être tout à la fois un instrument didactique apportant un important recueil d'informations - et une invite à prolonger soi-même un tel recueil à partir des orientations documentaires fournies -, une réunion d'analyses articulées entre elles et finalement un véritable essai incitant à la relecture et à la réflexion. Un point doit en particulier être souligné quant à la dimension pédagogique du livre : l'importance des apports de l'analyse longitudinale, sans laquelle l'approche de la non-qualification ne serait possible qu'au travers de la juxtaposition de données structurelles successives, sans représentation possible des parcours et donc sans compréhension des paradoxes que ceux-ci révèlent quant aux situations des individus et des groupes, appréciées de façon seulement synchronique. 
La reconnaissance de la pluralité des aspects de l'objet n'exclut pas la mise en relation synthétique à laquelle le lecteur est invité, tout en sachant bien qu'il ne peut s'agir que d'une " totalité ouverte» (Lefebvre, 1955), d'un work in progress perpétuellement inachevé... Cet exercice implicite est particulièrement tentant si on lit les unes après les autres, en suivant, les conclusions qui résument les apports des cinq premiers chapitres à la fin de chacun d'eux. On peut ainsi rapprocher les angularités des analyses opérées à partir de cinq entrées différentes pour, comme José Rose y invite le lecteur dès son introduction, atteindre le double préalable "d'affiner le diagnostic et de valider les interprétations" (p. 9).

En dépit de la pluralité des approches nécessaires, celle qui vient en premier comporte bien évidemment elle-même un caractère général et englobant, puisque le chapitre 1 de l'ouvrage se demande de quoi on parle quand on parle de "non-qualification ». Ce préalable qui, à certains égards pourrait sembler théorique et par là même un peu ardu - cueillant le lecteur en quelque sorte à froid !, n’en est pas moins méthodologiquement indispensable, pour cerner une catégorie protéiforme avant d'en examiner les illustrations concrètes et les représentations empiriques. L'aboutissement de cet acte premier de l'auteur vaut d'être cité, dans son apparente simplicité, justifiant au passage le choix d'un titre du livre sous forme de question : "S'il y a bien des emplois, des activités ou des personnes peu qualifiés, il n'y a pas, à proprement parler, de "non-qualification", car toute activité productive exige la mise en ceuvre d'un minimum de qualités» (p. 33).

Mais qu'est donc un phénomène qui perdure et qu'on peut observer, alors même qu'il ne devrait pas exister?

Le chapitre 2 reprend la question en l'appliquant aux emplois classés comme " non qualifiés " et en cherchant dans quels secteurs se trouvent ces ENQ et qui les occupe. De nombreuses corrélations sont convoquées pour répondre à ces interrogations. Elles aboutissent notamment à identifier cinq familles de trajectoires professionnelles caractérisant les destins possibles des personnes qui ont, à un moment ou à un autre, pu se trouver dans la population non qualifiée, en raison du caractère de l'emploi occupé (p. 50). Cette typologie permet d'établir que la progression des ENQ « est le résultat de processus à la fois économiques, politiques et sociaux» (p. 53), ce qui écarte ainsi, bien évidemment, l'idée qu' il n'y aurait qu'une seule catégorie de ces ENQ.

Un autre aspect de la pluralité des aspects du phénomène étudié est clairement illustré par le chapitre 3, qui aborde les formes, en particulier organisationnelle, du travail réputé non qualifié. Là encore, la diversité l'emporte sur ce qui serait un ensemble unique et homogène de traits généralement associés au TNQ. Ce chapitre est très important dans la mesure où il aboutit à l'idée que tout travail comporte de facto une certaine part de qualification qui rend impossible sa caractérisation comme travail intégralement sans qualification.

La troisième facette des analyses conduites porte sur les travailleurs « non qualifiés » euxmêmes. Elle est présentée dans le chapitre 4, dans lequel se retrouve un résumé des résultats classiques - mais dont le rappel est toujours nécessaire - sur la prégnance des facteurs 
pesant sur le devenir de ces travailleurs, à travers en particulier leurs caractéristiques scolaires et sociales. Par delà le constat du poids de l'accès au diplôme, il est mentionné que " ce critère n'est pas systématiquement déterminant car il y a aussi des non-diplômés sur des emplois qualifiés, et des diplômés sur des emplois non qualifiés" (p. 90). Cet élément va dans le sens d'une certaine hétérogénéité des ENQ et de la population qui les occupe.

La dernière approche angulaire proposée porte sur les relations entre les politiques publiques et la qualification, qui font l'objet du chapitre 5. Il en ressort que, non seulement les politiques publiques n'ont jamais pris la question des qualifications comme sujet frontal, mais encore qu'elles ont été caractérisées, depuis plus d'un quart de siècle, par une extrême fragmentation entre des politiques et des dispositifs portant sur des objets et des publics d'une extrême diversité, y compris à l'intérieur du champ des formations professionnelles, initiales et continues. Pour finir, l'accent peut être mis, en la matière, sur le fait, largement reconnu, que " les résultats de ces politiques ont été assez mitigés et nont en tout cas pas réduit la part des emplois non qualifiés ni accru les chances de reconnaissance des qualifications" (p.109).

\section{Un phénomène évolutif dont la permanence défie les politiques publiques}

Que pourraient donc être les éléments d'une politique publique tendant à obtenir une véritable baisse des ENQ et aussi à en faire sortir ceux qui s'y trouvent ?

Pour y répondre, les chapitres 6 à 8 proposent des éléments d'un caractère somme toute assez différent de ce qu'exposaient les chapitres 1 à 5 , le chapitre 8, terminal, allant même jusqu'à s'interroger sur ce que pourrait être effectivement la mise en œuvre d'" une politique de la qualification » dont le chapitre 7 avait seulement cherché à exprimer les objectifs formulables et les moyens d'action possibles à partir d'expériences récentes.

Le chapitre 6, d'abord, pourrait être considéré comme une sorte de transition entre les constats opérés et la recherche de solutions pour lutter contre le TNQ. Il ne propose pas à proprement parler des actions, mais met l'accent sur les perspectives de la " non-qualification ", ce qui conduit notamment à rappeler l'importance mais aussi la difficulté des démarches de prospective des qualifications.

Tous ceux qui ont participé, voici une douzaine d'années, au lancement des travaux du groupe de Prospective des Métiers et des Qualifications (PMQ), sous la présidence de Claude Seibel, au sein de ce qui était encore le Commissariat Général du Plan, se rappellent en effet du mélange d'enthousiasme et de scepticisme qui a entouré, à l'époque, une entreprise qui a fait date et s'est poursuivie par la suite sous une autre forme. Au total, comme l'écrit José Rose, "il n'est pas inutile de fournir des éléments de réflexion aux acteurs concernés et, éventuellement, des aides aux décideurs afin de leur permettre d'anticiper les évo- 
lutions et de faire évoluer leurs pratiques» (p. 112). Pour être délicat, l'exercice n'en est pas moins salubre, ne serait-ce que par ce qu'il est susceptible d'indiquer sur les tendances inscrites dans les changements présents lorsqu'on souhaite se porter au devant de l'avenir. Au moment même où les premiers travaux du groupe PMQ prenaient leur essor, il n'est pas indifférent de remarquer que les statisticiens mettaient en évidence, à contre-courant des idées alors à la mode, une double constatation particulièrement lourde : non seulement le nombre des emplois non qualifiés retrouvait, en 2001, son niveau de 1982, mais encore la part de l'emploi non qualifié dans l'emploi total paraissait s'être stabilisée au cours de la décennie des années 90 (Chardon, 2001).

La poursuite des travaux de prospective conduits par la suite a confirmé les tendances observées dans les faits : l'ENQ ne recule pas et joue notamment un rôle important dans les services, avec toutefois des situations d'une grande hétérogénéité.

Le chapitre $7 s^{\prime}$ interroge donc à bon droit sur les politiques conduites en faveur de la qualification. Il montre qu'elles ont essentiellement reposé, à différents moments, sur une logique d'action sur les facteurs constitutifs de la non-qualification, plutôt que sur une orientation générale, dont un exemple significatif a cependant été fourni par les préconisations du Centre d'analyse stratégique, dont le groupe "Travail non qualifié », animé par Paul Santelman et François Hubault, a su poser les éléments d'une véritable politique globale, articulant entre eux des éléments sectoriels (pp. 138-141). "Cette politique, [comme le remarque l'auteur], concerne tous les acteurs de la relation salariale, les employeurs comme le système éducatif ou les intermédiaires de l'emploi» (p. 139).

Il est évidemment tentant d'aller plus loin, ce qu'essaie de faire le chapitre 8 en cherchant ce que pourraient être "les facettes d'une politique de la qualification». Peut-on dire que ce chapitre laisse - mais pouvait-il vraiment en être autrement ? - un peu le lecteur sur sa faim en donnant l'impression qu'il prolonge en fait les expériences antérieures de lutte contre des aspects angulaires du phénomène visé, plutôt que de proposer des orientations réellement novatrices?

Mais ce léger reproche est facile à minorer si l'on veut bien considérer que l'auteur dispose sans doute lui-même des éléments d'un autre regard, plus radical à certains égards, qu'il pourrait porter sur le TNQ et les moyens de le faire reculer.

Pour cela, il suffit de remonter en arrière dans le livre. 


\section{Une question peut en cacher une ou plusieurs autres...}

En effet, la recherche de la nature du travail non qualifié, à travers les différentes approches de ses aspects, avait conduit l'auteur, dès la conclusion du chapitre 3 , à rechercher les axes d'une politique alternative, dont les deux chapitres 7 et 8 esquissent les principaux traits.

C'est finalement à ce point précis du livre que se situe peut-être son moment le plus fécond, celui qui pourrait porter en lui le plus fort potentiel de changement, plus fort sans doute que les actions multiples sur les différents problèmes liés à la question des accès à la qualification.

D’une certaine façon, suggère José Rose, toutes les caractéristiques et toutes les approches angulaires du TNQ n'ont pas les mêmes potentialités pour permettre de retourner la situation observée. Plus que sur l'emploi et sur le registre de la quantité, c'est sur le travail lui-même qu'il convient d'agir, en modifiant son approche en termes de qualité et de renversement de ce qu'une approche marxienne appellerait les rapports sociaux de production. Le point nodal de l'ouvrage consisterait donc à préconiser de partir avant tout d'une analyse du travail en mettant en évidence que c'est celui-ci qui définit le mieux la situation de tout travailleur, par delà les différences des modalités multiples de cette situation. En effet, "l'intensité du travail et la disponibilité de la personne sont des traits que l'on retrouve aussi bien dans les activités les plus hautement et les plus faiblement qualifiées ", ce qui découle corrélativement du fait qu' "une analyse fine des activités concrètes montre que dans chaque situation de travail, ily a une exigence et une mise en œuvre effective de compétences, de savoirs et de savoir-faire" (p. 72).

La reconnaissance des compétences des personnes et surtout le préalable de "la mise en visibilité de certaines d'entre elles " (ibid.) constituent les deux premières étapes nécessaires pour amorcer la disparition du TNQ. Mais pour y parvenir, encore faut-il, pour reprendre le terme précis qui figure au dos du livre, en $4^{\text {ème }}$ de couverture, que le travail dit non qualifié cesse d'être "dénié dans sa qualité". À ce stade, il est clair qu'il ne suffit pas de parvenir à un aménagement des nomenclatures des spécialités professionnelles ni même d'obtenir des révisions des classifications conduisant à la disparition de la «non-classification». En somme, dès l'introduction de l'ouvrage, rien d'autre n'était déjà affirmé avec force, à travers le constat de la "polarisation des qualifications" comme " consubstantielle à la division capitaliste du travail» (p. 9). Pour en finir avec le TNQ, il faut probablement aller plus loin encore en dévoilant "l'insoutenabilité sociale de la non-qualification", composante d'une action politique d'ensemble conduisant au " recul de la marchandise " (Harribey, op. cit., 2010). Mais ceci est une autre histoire... 


\section{Référence de l'ouvrage}

José Rose (2012), Qu’est-ce que le travail non qualifié ?, Paris, La Dispute, 180 p.

\section{Bibliographie}

Beduwe C. (2005), « Peut-on parler de relation formation-emploi au sein des emplois non qualifiés ? ", in Des formations pour quels emplois ?, La Découverte, pp. 348-365.

Belani P. (2007), "Subvention des emplois non qualifiés dans un marché du travail dual ", Revue économique, Vol. 58, 2007/3, pp. 693-702.

Chardon O. (2001), "Les transformations de l'emploi non qualifié depuis vingt ans », INSEE Première, $\mathrm{n}^{\circ} 786$, p. 1.

Harribey J.-M. (2010), "Eléments pour une économie politique de la soutenabilité fondée sur le recul de la marchandise ", Revue Française de Socio-Economie, n 06, pp. 31-46.

Lefevbvre H. (1955), « La notion de totalité dans les sciences sociales ", Cahiers internationaux de sociologie, Vol. 18, janvier-juin 1955, pp. 55-77.

Meda D., Vennat F. (eds.) (2005), Le travail non qualifié, La Découverte, collection « Recherche».

Tremblay D.-G. (dir.) (1996), «Innovation, technologie et qualification : une introduction », in Actes du 14 ${ }^{\mathrm{e}}$ Colloque de l'Association d'économie politique, pp. 11-17. 